\title{
Análisis del papel de las mujeres en la investigación contable desarrollada en el Departamento del Quindío desde una perspectiva de género
}

\author{
Analysis of the role of women in accounting research developed \\ in the department of Quindio from a gender perspective
}

Recibido: 02-03-2018 • Aprobado: 21-06-2018 • Página inicial: 157 - Página final: 180

Érika Julieth López Tejada*

\begin{abstract}
Resumen: este artículo es construido con el fin de ampliar la discusión sobre los campos de acción que involucran la disciplina y la profesión contable, profundizándose en las desigualdades y discriminaciones presentes por razones de género y reconociendo la participación femenina en el crecimiento de este campo de conocimiento; en ese sentido, se abordó la inclusión e intervención en la academia contable por parte de las mujeres, específicamente en el Departamento del Quindío. Tomando como base fundamental, la producción académica realizada, los cargos de liderazgo y la tendencia a la feminización de la profesión. Sin embargo, es evidente que a pesar de los cambios sociales y la gran presencia de mujeres profesionales contables, se siguen presentando casos de exclusión e inequidad, resultados de las concepciones culturales sobre la feminidad y la masculinidad que imperan en la sociedad actual.
\end{abstract}

Palabras clave: mujeres, género, investigación contable, estudios de género.
Abstract: This article is constructed with the purpose of broadening the discussion about the fields of action that involve the discipline and the accounting profession, deepening in the inequalities and discriminations present for reasons of gender and recognizing the participation of women in the growth of this field of knowledge, In this sense, the inclusion and intervention in the accounting academy by women was addressed, specifically in the Department of Quindío. Taking as a fundamental basis, the academic production carried out, the positions of leadership and the tendency to feminize the profession. However, it is evident that in spite of the social changes and the great presence of female accounting professionals, cases of exclusion and inequality continue to arise, results of the cultural conceptions of femininity and masculinity that prevail in today's society.

Keywords: Women, gender, accounting research and gender studies.

\footnotetext{
* Contadora Pública de la Universidad del Quindío, integrante del Consejo Estudiantil de Contaduría Pública de la Universidad del Quindío -COESCO-.

erikaj-lopezt@outlook.com.

Enlace ORCID: https://orcid.org/0000-0002-8215-6388.
} 


\title{
Analyse du rôle des femmes dans la recherche comptable développée dans le Département de Quindío d'une perspective de genre
}

\begin{abstract}
Résumé: cet article est construit dans le but d'élargir la discussion sur les champs d'action impliquant la discipline et la profession comptable, approfondissant les inégalités et les discriminations présentes pour des raisons de genre et reconnaissant la participation des femmes dans la croissance de ce champ de connaissance, En ce sens, l'inclusion et l'intervention dans l'académie de comptabilité par les femmes a été abordée, en particulier dans le département de Quindío. Prendre comme base fondamentale, la production académique réalisée, les positions de leadership et la tendance à féminiser la profession. Cependant, il est évident qu'en dépit des changements sociaux et de la grande présence des femmes comptables professionnelles, des cas d'exclusion et d'inégalité continuent à apparaître, résultats des conceptions culturelles de la féminité et de la masculinité qui prévalent dans la société actuelle.
\end{abstract}

Most-clés: femmes, genre, recherche comptable, études de genre.

\section{Análise do papel das mulheres na pesquisa em contabilidade desenvolvida no Departamento do Quindío a partir de uma perspectiva de gênero}

\begin{abstract}
Resumo: este artigo é construído com o objetivo de ampliar a discussão sobre os campos de ação que envolvem a disciplina e a profissão contábil, aprofundando as desigualdades e discriminações presentes por razões de gênero e reconhecendo a participação das mulheres no crescimento dessa área do conhecimento, Nesse sentido, a inclusão e intervenção na academia de contabilidade pelas mulheres foi abordada, especificamente no departamento de Quindío. Tomando como base fundamental, a produção acadêmica realizada, as posições de liderança e a tendência a feminizar a profissão. No entanto, é evidente que, apesar das mudanças sociais e da grande presença de profissionais contábeis do sexo feminino, continuam a surgir casos de exclusão e desigualdade, resultados das concepções culturais de feminilidade e masculinidade que prevalecem na sociedade atual.
\end{abstract}

Palavras-chave: mulheres, gênero, pesquisa contábil, estudos de gênero. 


\section{Introducción}

Los estudios de género nacen como resultado de los aportes e investigaciones realizadas desde el enfoque del feminismo académico. Entendiendo que este tuvo sus inicios en las instituciones universitarias, y que se ha ido incluyendo en las investigaciones científicas de las diferentes disciplinas naturales, sociales y humanas. La contabilidad no es ajena a la realización de este tipo de estudios, teniendo en cuenta que existen diversidad de autores/as que han problematizado los diferentes contextos en los cuales se encuentra presente la disciplina y la profesión contable como: Cooper (2001); Haynes (2008a, 2008b y 2016); entre otras. No obstante, el desarrollo de este tipo de investigaciones en el contexto colombiano no es abordado de forma significativa, por lo cual se encuentran pocas investigaciones y aportes referentes a la participación femenina en los diferentes contextos contables y los enfoques de género; en ese sentido vale resaltar los trabajos realizados por Acosta, Patiño, Valero y Díaz (2015); Calvo (2010); Chamorro (2015); León y Naranjo (2011) y Rodríguez (2014), los cuales poseen este tipo de visiones. Sin embargo, el propósito de este artículo es identificar el papel de las mujeres en el desarrollo de la investigación contable del Departamento del Quindío e incentivar la realización de estudios futuros sobre estas temáticas con el propósito de aportar al desarrollo del campo de conocimiento contable.

En ese sentido, este documento tiene como objetivo realizar un acercamiento sobre la importancia de los estudios de género en el ámbito contable y dar reconocimiento a las mujeres que realizan actividades investigativas en estos espacios. Este artículo se divide en las siguientes secciones: en primer lugar, se exponen los conceptos fundamentales empleados en la realización de la investigación. En segundo lugar, se abordan datos históricos sobre la presencia de las mujeres en las diversas áreas contables, exponiendo al final su presencia en los grupos de investigación contable del Departamento del Quindío. En tercer lugar, se muestra la metodología empleada en la investigación. En cuarto lugar, se presentan y analizan los resultados obtenidos, manifestándose en estos una aproximación teórica a la realización de estudios de género en contabilidad; seguidamente se describen y clasifican las publicaciones de las revistas seleccionadas por el género de los autores (as), posteriormente, se expresan algunas apreciaciones sobre estas temáticas dadas por las mujeres entrevistadas, con el propósito de darle una mayor profundidad a la investigación. Por último, se ostenta la discusión y algunas conclusiones referentes a los resultados presenciados en el desarrollo de este trabajo. Con relación a lo planteado anteriormente, este documento se expresa como un llamado a la inclusión de estas temáticas en el ejercicio de la investigación contable. 


\section{Metodología}

La metodología utilizada para el desarrollo de esta investigación se caracterizá por ser cualitativa, permitiendo el análisis de la evolución académica y contextual del papel de las mujeres en la investigación contable realizada en el Departamento del Quindío. Así mismo, se emplearon diferentes fuentes para la recolección de información documental y empírica como: libros, artículos científicos, entrevistas y datos estadísticos descriptivos. Esto, con el propósito de interpretar y comprender el contexto en el cual se encuentra implícito el accionar de las mujeres en el campo de conocimiento contable.

\section{Conceptos fundamentales}

Se concibe para la realización de este proyecto que el sexo corresponde a las características físicas y biológicas que definen las diferencias entre hombres y mujeres. En su contraste se define al género como los rasgos y comportamientos atribuidos a cada uno de los sexos, entendiendo que, Mujer $=$ Femenino y Hombre=Masculino. Considerándose así que estos conceptos han sido aceptados de forma cultural en diversas comunidades y se han convertido en las definiciones dominantes sobre la feminidad y la masculinidad. Al lado de esto los modelos de conducta establecidos han sido garantes en la construcción de desigualdades entre hombres y mujeres que han favorecido a unos y perjudicado a otras; donde se les asignó a los varones la oportunidad de ejercer diversas actividades relacionadas con la vida pública (el trabajo, la participación política, entre otras) y se limitó el accionar de las mujeres direccionándolas hacia las labores domésticas, llamando a esto la vida privada.

Con base en lo afirmado anteriormente se entiende el feminismo como un movimiento social, político y académico que se ha encargado de identificar diversas problemáticas relacionadas con la desigualdad y la discriminación, con el propósito de buscar soluciones e interpretaciones sobre las mismas; por tal razón, el género se ha convertido en una variable de investigación científica y una categoría de análisis crítico en el desarrollo de diferentes investigaciones. Estos sucesos son resultados del ejercicio de la investigación feminista; actividades que iniciaron en las universidades por influencia de las mujeres activistas que ingresaron a estas instituciones. Este tipo de investigación se ha enfocado en el estudio de las distintas situaciones sociales, económicas y políticas que manifiestan las mujeres. Ha tomado como punto de partida y de referencia en las discusiones científicas la subjetividad femenina (Reigraf \& Aulenbacher, 2012) y cuestiona la supuesta neutralidad de género presente en las teorías e investigaciones científicas, exponiendo que estas han sido 
construidas desde un enfoque de parcialidad o visión masculina, a las cuales se les ha otorgado la generalidad. Por otro lado, Bartra (2012) asevera que, "el sexo de quien lleva a cabo la investigación puede condicionar todo el proceso de la investigación y es importante metodológica y epistemológicamente que eso se tome a consideración" (p.74).

La investigación feminista y de género se ha enfocado en la crítica de las teorías científicas dominantes, desde las ciencias sociales, humanas y naturales, donde se ha ido demostrando que la validez otorgada a las teorías y metodologías científicas no es general; teniendo en cuenta que estas demuestran la localización social de los investigadores, sus intereses y contextos vitales; por lo tanto, se ha revelado que los procesos de realización científica se han basado en las diferencias existentes entre los sexos, hecho que influye en los resultados (Reigraf \& Aulenbacher, 2012). A esto se añade que las teorías del conocimiento han sido construidas desde una visión androcéntrica y sexista del mundo, manifestando mayor interés hacia los fenómenos que los hombres consideran importantes; por esto, la participación de las mujeres se ha encontrado en las sombras de las teorías, los métodos y las investigaciones científicas (Blazquez, 2012). El hecho de considerar a los varones como el centro de la humanidad, influenció en la creación del conocimiento; como resultado de esto se dejó de lado la participación femenina; por lo mencionado anteriormente, se vio la necesidad de contribuir en la construcción y reconstrucción de los acontecimientos importantes, con el propósito de identificar el papel de las mujeres en el desarrollo de los mismos; en ese aspecto, se direccionó hacia la cimentación de un ideal científico particular e independiente; es así como la investigación feminista se caracteriza por contener una metodología no sexista y no androcéntrica (Bartra, 2012).

La perspectiva de género reconoce que existen ideas y creencias basadas en la separación de actividades por razones de sexo/género, lo que ha creado una participación desigual dentro de las instituciones sociales, políticas y económicas. En este sentido se busca analizar las formas de subordinación de las mujeres y la opresión ejercida por los hombres hacia ellas como resultado de las creencias patriarcales existentes (Facio \& Fries, 1999) (Ríos, 2012). Así mismo, vale afirmar que los estudios con perspectiva de género se han enfocado principalmente en las condiciones y situaciones de las mujeres, siendo estas mayormente oprimidas y marginalizadas por las ideologías patriarcales; pero sobre los hombres también se han venido desarrollando este tipo de estudios; un ejemplo de ello es la discusión actual sobre las nuevas masculinidades, entendiendo también que ellos se ven sometidos a diversas problemáticas sociales como: la pobreza, el desempleo, entre otras. 


\section{Contexto Loose E.}

Los estudios con enfoque de género cimientan el ideal sobre la igualdad que debe existir entre hombres y mujeres; sin embargo, esta igualdad no se trata de llevar a las mujeres a la categoría de hombres, porque se estaría entrando en la lógica patriarcal de que lo humano es lo considerado masculino; al contrario, se parte del reconocimiento y aceptación de las diferencias entre los sexos, teniendo en cuenta que poseen las mismas capacidades y características intelectuales para desarrollar diversidad de actividades que demanden altos grados de responsabilidad.

Estos pensamientos han surgido como resultado del trabajo académico y político de mujeres intelectuales organizadas, con la intención de incorporar el punto de vista femenino a la comprensión de los diversos procesos sociales. Han realizado un aporte de gran importancia en la generación de conceptos y metodologías, con el fin de producir nuevos conocimientos, entre estos el de género (Todaro \& Rodríguez, 2001); de este modo, se pretende percibir las situaciones en las cuales se encuentra inmersa la sociedad, y cuestionar las teorías e investigaciones científicas tradicionales, generando análisis alternativos sobre los acontecimientos sociales en los cuales se involucra la participación entre hombres y mujeres.

\section{Contexto}

\section{Las mujeres y la profesión contable}

En Colombia en 1933 les fue otorgado el derecho a las mujeres de ingresar a la educación superior, donde estas en primera instancia decidieron formase en carreras consideradas culturalmente como femeninas o feminizadas, por su relación con las concepciones y estereotipos sobre las actividades que las mujeres debían realizar, siendo estas: la enfermería, el trabajo social, la comunicación social, las licenciaturas, entre otros estudios relacionadas con el cuidado del otro. Más adelante se fueron incorporando en el estudio de las ingenierías, ciencias políticas, ciencias naturales, economía, administración, contaduría, entre otras carreras consideradas de exclusividad masculina (Bonilla, 1993).

No obstante, la enseñanza de la contabilidad en Colombia se originó por la necesidad de poseer empleados capacitados, que pudieran responder a las necesidades del entorno, en plena época de la industrialización (Cubides, 1999; Calvo, 2010). Sin embargo, el personal que recibía este tipo de formación debía ser masculino, porque su ejercicio se encontraba ligado al mundo empresarial y de los negocios, actividades que demandan un alto grado de responsabilidad; 
por las creencias sobre las incapacidades intelectuales y físicas de las mujeres a estas no les era permitido desarrollarlas. Por consiguiente, en los inicios de la enseñanza de la Contaduría Pública como profesión, según Calvo (2010), existían grupos muy pequeños y la participación femenina era mínima; por ello, Juan Jacobo Pavajeau expresa que los grupos de estudiantes eran muy pequeños y mayoritariamente masculinos porque las mujeres aún no se habían tomado las universidades; en los grupos de contadores empíricos tampoco existía una amplia participación femenina; igualmente no había presencia de ellas en el ejercicio de la docencia (Calvo, 2010).

No se pretende desconocer que las mujeres por su parte se han visto relacionadas con el ejercicio contable, comprendiendo que sus funciones se han direccionado hacia la administración del hogar y la familia; es así como el papel de la mujer frente al hogar llevaba consigo en algunas ocasiones la teneduría de las cuentas como ejercicio importante de la administración doméstica (Méndez, 2010). Lo anterior conduce a que las actividades domésticas le dieron el conocimiento empírico de las cuentas, dado que las mujeres han realizado y conservado las prácticas contables direccionadas a la conservación del capital. En consecuencia, el acceso a la educación superior les dio la oportunidad de incluirse en el aprendizaje de lo contable, espacios en los cuales las mujeres fueron aumentando su participación e incorporándose en las prácticas del ejercicio profesional; en ese sentido la oferta de formación fue creciendo mediante escuelas y centros educativos que ofrecían este tipo de capacitación contable (Méndez, 2010). Pero el campo contable en todos sus contextos seguía siendo dominado por varones "y desde los centros de enseñanza se desanimaba a las mujeres para que no emprendieran la carrera contable, en vista de las dificultades con las que se encontrarían" (Méndez, 2010, p.97).

En el contexto contable universitario actual, se observa una amplia participación femenina tanto en el aprendizaje como en la enseñanza; comparando esta situación con años anteriores en los cuales estas actividades eran predilectas para los varones, siendo esta la causa de la existencia de la dominación masculina en lo académico, profesional y laboral contable. Considérese aquí que los cambios sociales presenciados en campo de conocimiento contable no garantizan que las concepciones culturales sobre los géneros y el desarrollo profesional hayan cambiado; resulta entonces que la oferta de profesionales contables posee una tendencia a la feminización, pero, el control sobre la demanda de la misma continúa en manos masculinas (León y Naranjo, 2011). Siendo los puestos directivos y profesionales directamente relacionados con el género, estos en su mayoría son ejercidos por varones y los cargos técnicos contables o no 


\section{Contexto Lcoose E}

contables son ocupados en gran parte por mujeres (León y Naranjo, 2011). Rodríguez (2014) asevera que la práctica contable consta de actividades mecánicas y repetitivas que necesitan de la disciplina, el orden y la fidelidad del personal, con el propósito de elaborar y llevar un manejo apropiado de los hechos económicos y financieros. Estas actividades se encuentran directamente relacionadas con los estereotipos impuestos sobre la feminidad, convirtiéndose las mujeres en el recurso humano necesario para la realización de las mismas, teniendo en cuenta que se caracterizan por ser: disciplinadas, fieles, ordenadas, pulcras y delicadas.

Ahora bien, con el fin de llevar estas concepciones al Departamento de Quindío, se solicitó en la Junta Central de Contadores, información sobre el número de personas registradas en este Departamento en el periodo 2010-2015 de lo cual se presentaron los siguientes datos.

Tabla 1.

Clasificación de los y las profesionales contables del Departamento del Quindío por género

\begin{tabular}{lcccc}
\hline Género & $\begin{array}{c}\text { Universidad del } \\
\text { Quindío }\end{array}$ & $\begin{array}{c}\text { Universidad la Gran } \\
\text { Colombia (Armenia) }\end{array}$ & Total & \% Total \\
\hline Femenino & 583 & 25 & 608 & $71 \%$ \\
\hline Masculino & 240 & 4 & 252 & $29 \%$ \\
\hline Total & 823 & 29 & 860 & $100 \%$ \\
\hline
\end{tabular}

Junta Central de Contadores, 2016.

Es evidente que el número de personas de género femenino es mayor al masculino en un 71\%; esto quiere decir que en el Departamento del Quindío existe un gran número de mujeres capacitadas y formadas para ejercer la profesión contable. Observándose entonces un resultado positivo en cuanto a la presencia de las mujeres en el campo del conocimiento contable, de lo cual se considera necesario indagar la situación expuesta con la intención de identificar si las concepciones culturales sobre las capacidades intelectuales de las mujeres y las percepciones sobre la feminidad han cambiado. 


\section{Las mujeres y la investigación contable}

Siendo principalmente del ejercicio investigativo la función de construir conocimiento, esta actividad en primera instancia fue asociada a la masculinidad, una labor profundizada en el desarrollo del capitalismo en el marco de la modernidad científica sexista (Bonilla, 1993). Dentro de este contexto las mujeres no eran incluidas en la ejecución de estas actividades e igualmente no eran reconocidas como investigadoras. Aunque las acciones femeninas en busca del reconocimiento como mujeres capaces de realizar las mismas funciones que los hombres, y su interés por adquirir nuevos conocimientos y participar en la construcción de los mismos, las ha ido incluyendo en actividades académicas de gran importancia; de ello resulta que gran parte de las concepciones que se poseían sobre las mujeres han ido cambiando. Una vez señalada esa distinción es importante reconocer que la dominación masculina y la subordinación femenina siguen vigentes en el imaginario individual y colectivo de hombres y mujeres, el cual es evidente en sus comportamientos y actitudes, siendo cierto que en la actualidad se han generado algunos cambios y transformaciones (Cabral y García, s.f).

Apréciese entonces que el desarrollo de la investigación científica en el campo del conocimiento contable no es ajena a este tipo de concepciones, comprendiendo que se ha caracterizado por ser una actividad masculina. En la actualidad, existe diversidad de mujeres que se encuentra ejerciendo su profesión en estos espacios; tanto así, que están ocupando cargos de liderazgo en los grupos de investigación. Teniendo en cuenta que esta investigación se realiza en el Departamento del Quindío, a continuación se muestran los grupos de investigación contables presentes en este sector, avalados por Colciencias según los resultados de la convocatoria 737 de 2015, dados a conocer en mayo de 2016:

Tabla 2.

Grupos de investigación contable presentes en el Departamento del Quindío avalados por Colciencias

\begin{tabular}{lccc}
\hline \multicolumn{1}{c}{ Grupo de investigación } & $\begin{array}{c}\text { Líder del grupo de } \\
\text { investigación }\end{array}$ & Institución & $\begin{array}{c}\text { Año de } \\
\text { creación }\end{array}$ \\
\hline $\begin{array}{l}\text { Gestión contable, financiera y } \\
\text { tributaria (GECOFIT) }\end{array}$ & $\begin{array}{c}\text { Marlon David García } \\
\text { Jiménez }\end{array}$ & $\begin{array}{c}\text { Universidad la Gran } \\
\text { Colombia- Armenia }\end{array}$ & 2009 \\
\hline $\begin{array}{l}\text { Grupo de Investigación en } \\
\text { Responsabilidad Social (GRS) }\end{array}$ & $\begin{array}{c}\text { Isabel Cristina Londoño } \\
\text { Franco }\end{array}$ & $\begin{array}{c}\text { Escuela de } \\
\text { Administración y } \\
\text { Mercadotecnia }\end{array}$ & 2007 \\
\hline
\end{tabular}




\section{Contexto Looes E.}

\begin{tabular}{|c|c|c|c|}
\hline Grupo de investigación & $\begin{array}{l}\text { Líder del grupo de } \\
\text { investigación }\end{array}$ & Institución & $\begin{array}{l}\text { Año de } \\
\text { creación }\end{array}$ \\
\hline $\begin{array}{l}\text { Grupo de Investigación en } \\
\text { Gerencia y Emprendimiento } \\
\text { (GIGA) }\end{array}$ & $\begin{array}{l}\text { Edwin Tarapuez } \\
\text { Chamorro }\end{array}$ & $\begin{array}{c}\text { Universidad del } \\
\text { Quindío }\end{array}$ & 2009 \\
\hline $\begin{array}{l}\text { Grupo de Investigación } \\
\text { en Derecho Tributario } \\
\text { Comparado y Desarrollo } \\
\text { Empresarial de la Universidad } \\
\text { Del Quindío (GEDUQ) }\end{array}$ & $\begin{array}{l}\text { Constanza Loreth } \\
\text { Fajardo Calderón }\end{array}$ & $\begin{array}{c}\text { Universidad del } \\
\text { Quindío }\end{array}$ & 2012 \\
\hline $\begin{array}{l}\text { La Función Financiera } \\
\text { en las Organizaciones del } \\
\text { Departamento del Quindío }\end{array}$ & $\begin{array}{c}\text { Ana Gladys Torres } \\
\text { Castaño }\end{array}$ & $\begin{array}{c}\text { Universidad del } \\
\text { Quindío }\end{array}$ & 2004 \\
\hline
\end{tabular}

Elaboración propia, 2016.

Se observa en la Tabla 2 que de los cinco grupos de investigación seleccionados, tres son liderados por mujeres y dos por hombres. Para el desarrollo de esta investigación se han tomado los datos de los Gruplac desde el año 2004, que es la fecha de creación del primer grupo, hasta el año 2015. Con el fin de observar y clasificar los productos realizados por los grupos según la categorización de Colciencias y por el género de las autoras y autores.

\section{Los estudios de mujer y género en Colombia}

El origen de los estudios de género y ciencia se da en los Estados Unidos en la década de los 70's; por consiguiente, en los años 80's empiezan a desarrollarse en Europa (Nuño, 2000). Comenzaron entonces a comprenderse este tipo de estudios como las críticas feministas de las ciencias sociales y naturales, independientemente de que fueran realizados por hombres o mujeres (Nuño, 2000). En Colombia, los estudios de género se originaron en los años 90's en la Universidad Nacional de Colombia, siendo estos promulgados por los movimientos feministas existentes en la época que ingresaron al campo académico. Por ello, se obtuvo una amplia participación en la elaboración de la Constitución Política de 1991, donde se garantizó la igualdad de derechos entre hombres y mujeres. Actualmente en Colombia existen diversas autoras reconocidas por su labor en desarrollo de los estudios de género, como: Magdalena León, Luz Gabriela Arango, Florence Thomas y Yolanda Puyana (Acosta, Patiño, Valero y Díaz, 2015).

La perspectiva de género se introdujo en las diferentes disciplinas sociales, humanas y naturales, de las cuales la contabilidad no ha sido ajena. Esto con el 
fin de identificar las desigualdades existentes entre los sexos por influencia de las ideologías dominantes científicas y socioculturales, y de este modo, generar explicaciones alternativas sobre las problemáticas sociales en las cuales se encuentran inmersos los géneros, la disciplina y la profesión contable.

A la investigación contable con perspectiva de género desarrollada en Colombia no se le ha dado una importancia reveladora; a causa de ello, en la búsqueda documental de publicaciones nacionales solo se encontraron dos artículos sobre estos tópicos en el periodo 2010-2015. En la revista Contaduría el artículo titulado: "Influencia de los estereotipos de género en el área contable en las grandes empresas de Medellín", con la autoría de Giraldo, Jaramillo, Torres y Gómez, del año 2011; y en la revista Activos el artículo que titula: "Las problemáticas de género: una introducción para su aplicación en estudios de contabilidad", escrito por Acosta, Patiño, Valero y Díaz, en el año 2015. A diferencia de las publicaciones encontradas en revistas internacionales, donde se manifiesta una participación más amplia referente a estos contenidos; en ese sentido vale afirmar que los estudios de género y la implementación de las teorías feministas en la contabilidad no son temáticas nuevas en el campo de conocimiento contable, pero a estas no se les ha dado una categoría significativa en el desarrollo de la disciplina.

\section{Resultados}

\section{Los estudios de género en la investigación contable}

El enfoque de género se encuentra directamente relacionado con el pensamiento feminista y se ha transformado en la forma de expresión de las mujeres en la política, la academia y la sociedad. El desarrollo de estudios con perspectiva de género en contabilidad ha sido generado por diversas autoras (es), de los cuales, se han establecido discusiones sobre las problemáticas sociales donde se encuentra inmersa la disciplina y la profesión. Sin embargo, Haynes (2016) afirma que, el concepto de género se encuentra relacionado con hombres y mujeres, pero muchas investigaciones con este enfoque se centran específicamente en la posición de las mujeres, teniendo en cuenta que ellas han sido sometidas a mayores casos de discriminación y opresión por las condiciones patriarcales. A causa de ello, los documentos investigativos sobre estas temáticas en contabilidad se enfocan en los contextos y problemáticas de las mujeres por ser más propensas a la exclusión y opresión en los espacios contables. En ese aspecto Cooper (2001) asevera que muchas de las investigaciones realizadas en contabilidad se han enfocado en la satisfacción de las necesidades de los grupos dominantes, donde el lenguaje científico se ha manifestado con un 
aspecto de neutralidad, pero dejando de lado las diversas problemáticas sociales en las cuales se involucra la disciplina y la profesión. Deduciendo que las investigaciones realizadas por parte de la corriente principal en contabilidad no se hacen responsables de los hechos negativos que puedan ocasionar en la sociedad; ignorando así las divergencias sociales y económicas que afectan las diferentes comunidades en estado de marginalidad.

Las investigaciones sobre género realizadas en el contexto contable se han enfocado especialmente en el ingreso de las mujeres a la profesión y en los aportes que ellas han construido para los avances de la misma (Cooper, 2001). Este tipo de estudios permiten ampliar el campo de acción de la contabilidad abordando diferentes temáticas como el clasismo, racismo, sexismo, entre otros casos discriminatorios, permitiendo la utilización de variables que incluyen las indagaciones sobre la identidad (género, sexo, etnia, clase social, etc.). Por esto se propende por la obtención de interpretaciones alternativas de los resultados investigativos que fomenten la inclusión de los sectores marginalizados y minoritarios de la sociedad; partiendo así de la idea de generar nuevos conocimientos que problematicen los puntos de vista tradicionales y busquen soluciones a estas problemáticas, teniendo en cuenta que este tipo de trabajos multidisciplinarios permiten la consecución de nuevos métodos con temáticas de género y la creación de nuevos conceptos, con el fin de expandir los márgenes de la investigación contable con perspectiva de género.

Vale afirmar que este tipo de enfoques son aplicables en el campo del conocimiento contable, partiendo de la imagen en la cual la contabilidad posee género; en ese sentido esta se encarga de reforzar los estereotipos sobre la feminidad y la masculinidad (Haynes, 2008a; 2008b). Así mismo, se ha caracterizado por revelar las opresiones y discriminaciones aceptadas socialmente, proporcionando una nueva visión sobre los roles de género y la generización de la contabilidad (Haynes, 2016).

La investigación feminista y de género realizada en el mundo contable logra identificar el efecto de la contabilidad en los diferentes contextos globalizados y particulares en la revelación de las diferentes clases de manifestación de la opresión y las desigualdades; igualmente, vela por el reconocimiento de las formas de resistencia organizadas que se manifiestan frente a estos sucesos.

Por lo visto, la realización de investigación con influencia de las teorías feministas se ha involucrado en las diferentes áreas del conocimiento, entre estas la contable, lo que ha permitido enfocarse en la creación de visiones e interpretaciones alternativas de las diferentes problemáticas sociales, y muestran 
las formas de opresión y discriminación por razones de sexo, género, raza, entre otras. En ese aspecto, se profundiza en la creación de teorías, metodologías e investigaciones que eliminen los sesgos discriminatorios, con el propósito de construir conocimiento que se adapte a las necesidades sociales actuales.

\section{Publicaciones de revistas realizadas entre el periodo 2010-2015}

En primer lugar se seleccionaron cinco revistas contables con el propósito de identificar el número de publicaciones realizadas por mujeres en el periodo 2010-2015, estas son: a) Cuadernos de Contabilidad, b) Activos, c) Lúmina, d) Contaduría, y, e) Internacional Legis de Contabilidad y Auditoría. Siendo esta información clasificada en cuatro categorías: 1) Femenino, 2) Masculino, 3) Femenino y Masculino, y, 4) No aplica; teniendo en cuenta que la categoría tres (masculino y femenino) corresponde a las publicaciones realizadas con participación de ambos géneros y la categoría cuatro (no aplica) hace referencia a las publicaciones realizadas por entidades gubernamentales como: La Junta Central de Contadores, El Consejo Técnico de la Contaduría, las Superintendencias, entre otras. De lo cual se obtuvieron los siguientes resultados:

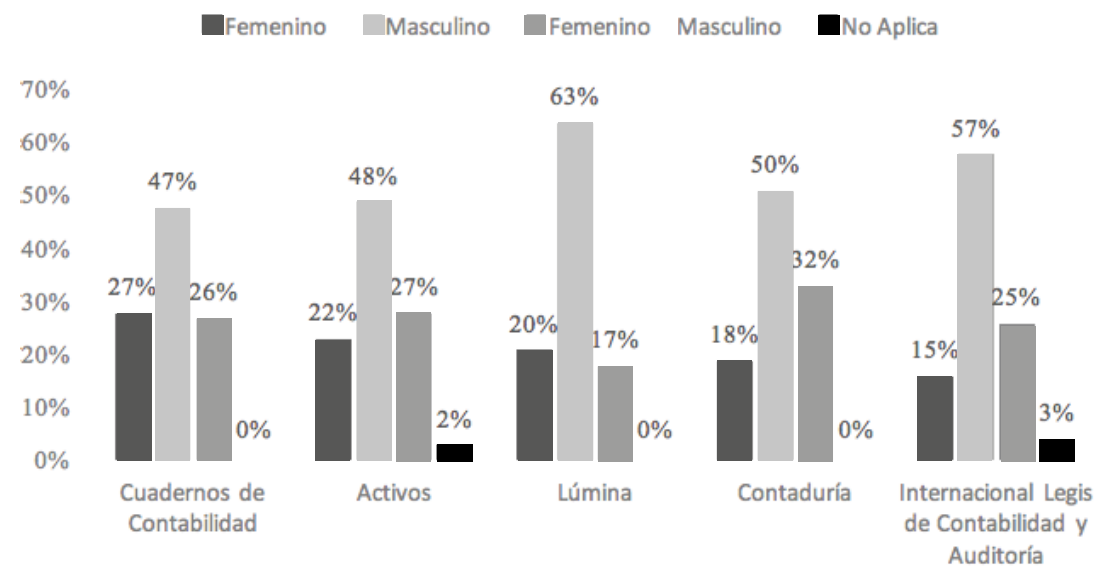

Figura 1. Consolidado de las revistas por género

Elaboración propia, 2016.

La Figura 1 muestra el porcentaje de publicaciones en las revistas distribuido por género, entendiendo que las publicaciones de autoría femenina se encuentran entre $15 \%$ y $27 \%$, las publicaciones masculinas entre el $47 \%$ y el $63 \%$, por último, las publicaciones realizadas con participación de ambos géneros se encuentran entre el $17 \%$ y el $32 \%$. En comparación con lo anterior, 


\section{Contexto Lopoes E.}

la Figura 2 expone el porcentaje de publicaciones por cada año consultado, de lo cual se afirma que las publicaciones femeninas realizadas en este periodo se encuentran entre en $17 \%$ y $29 \%$, las masculinas entre el $47 \%$ y $53 \%$, por último, las publicaciones realizadas con participación de ambos géneros se encuentran entre el $21 \%$ y $35 \%$.

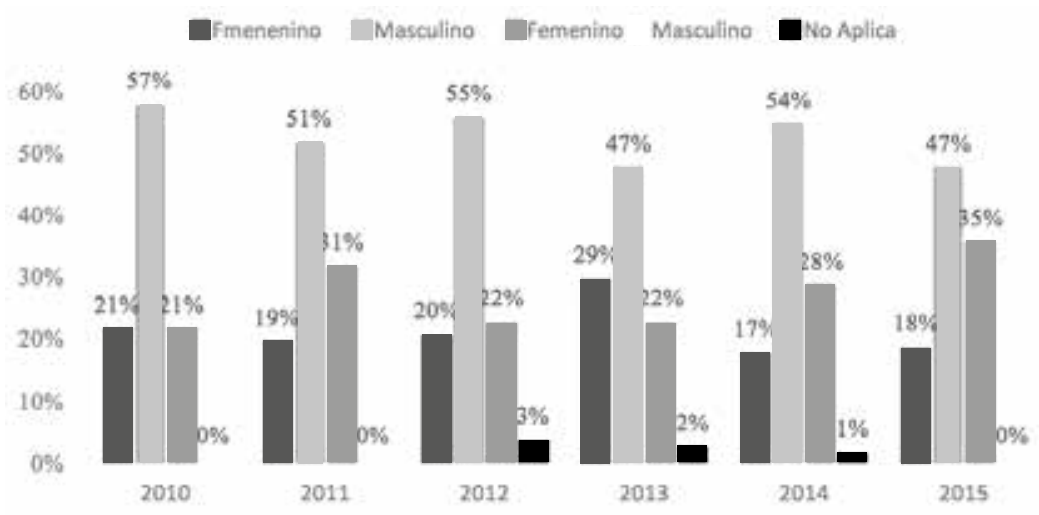

Figura 2. Consolidado de las revistas por año

Elaboración propia, 2016.

Se asevera entonces que de las revistas seleccionadas el porcentaje de las publicaciones femeninas no alcanza el $40 \%$, en comparación de las publicaciones masculinas que sobrepasan el $50 \%$ de los datos. Es importante reconocer que a pesar de que la participación femenina es baja, se está manifestando un cambio en los procesos de publicación, ya que existe un gran número de mujeres pensándose la disciplina y la profesión contable. Hecho por el cual es pertinente aseverar que los hombres siguen dominando el campo de la investigación contable, pero las mujeres también se encuentran realizando este tipo de ejercicios investigativos y de producción académica.

\section{Producción académica realizada en los grupos de investigación seleccionados}

En segundo lugar se tomó la información en los Gruplac de los cinco (5) grupos de investigación seleccionados para esta investigación. De lo cual según la gráfica 3 se obtuvo que del número total de integrantes de los grupos el 67\% corresponde a personas del género femenino y el $33 \%$ a personas del género masculino, como se muestra seguidamente: 


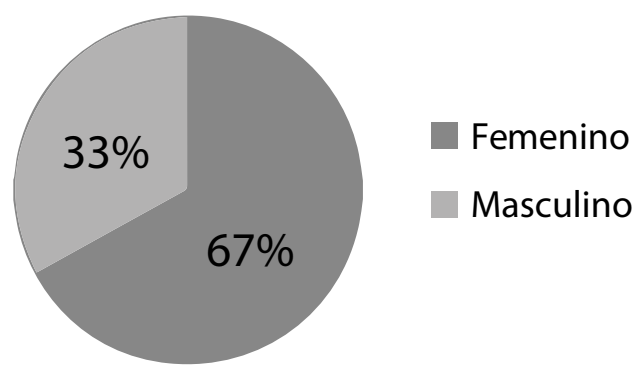

Figura 3. Integrantes de los grupos de investigación contable por género

Elaboración propia, 2016.

A saber, la Figura 4 muestra que el porcentaje de producción femenina en los grupos de investigación seleccionados se encuentra entre el $42 \%$ y el $91 \%$, la producción masculina oscila entre el $5 \%$ y el $47 \%$, y, por último, la producción realizada con participación de ambos géneros se encuentra entre el 0 y $22 \%$. Es evidente que en el Departamento del Quindío gran parte de la producción académica realizada en los grupos de investigación contable son de autoría femenina, evidenciando así una amplia participación de género femenino en el desarrollo de este tipo de actividades.

Demenino Masculino EFemenino Masculino

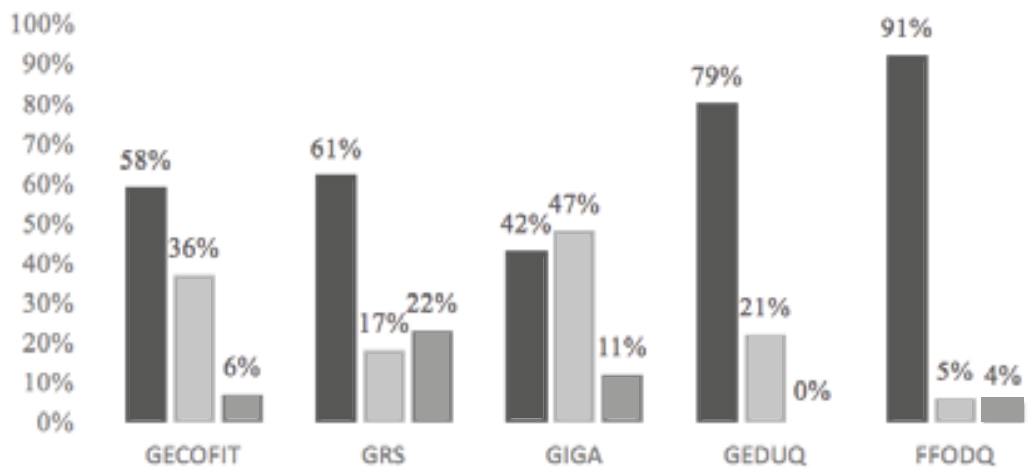

Figura 4. Producción de los grupos por género

Elaboración propia, 2016.

Seguidamente, se clasificó esta información según la categorización de los productos dada por Colciencias en el "Modelo de medición de grupos de 
investigación, desarrollo tecnológico o de innovación y de reconocimiento de investigadores del sistema nacional de ciencia, tecnología e innovación, año 2015 ", la cual se expresa a continuación:

- Generación de nuevo conocimiento: son aportes al estado del arte o conocimiento, que han sido validados mediante la discusión científica, mediante el ejercicio de actividades investigativas, como: artículos de investigación, libros resultados de investigación, entre otros (Colciencias, 2015).

- Desarrollo tecnológico e innovación: son productos que generan impacto en el desarrollo económico y buscan una transformación social mediante la creación de métodos, herramientas e ideas, como: legislaciones, regulaciones, normas, productos empresariales, consultorías, entre otros (Colciencias, 2015).

- Productos resultados de apropiación social del conocimiento: son procesos y prácticas sociales que buscan el intercambio de conocimientos, como: participación ciudadana en $\mathrm{CTeI}^{1}$ y creación; estrategias pedagógicas para el fomento de la CTeI; comunicación social del conocimiento; circulación de conocimiento especializado; y reconocimientos (Colciencias, 2015).

- Productos de Actividades relacionadas con la formación de recurso humano para CTeI: Actividades relacionadas con el servicio de formación de nuevos investigadores (as), como: asesorías en tesis de grado de maestría, trabajo de grado de pregrado; realización de proyectos de investigación y desarrollo; proyectos de investigación-creación; proyecto de investigación, entre otras (Colciencias, 2015).

Teniendo en cuenta lo mencionado con anterioridad, la Figura 5 expone que del total de los productos realizados por los grupos de investigación seleccionados, la participación femenina en la elaboración de estos se encuentra entre el $40 \%$ (Apropiación social del conocimiento) y el 82\% (Formación de recurso humano para $\mathrm{CTeI}$ ), pero teniendo en cuenta que en todas las categorías existe gran presencia de este género. Así, pues, que la participación masculina se encuentra entre el 11\% (Desarrollo tecnológico e innovación) y el 45\% (Apropiación social del conocimiento), y, por último, la realización de productos por medio de la participación de ambos géneros se encuentra entre el 1\% (Formación de recurso para CTeI) y 25\% (Generación de nuevo conocimiento).

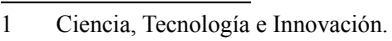




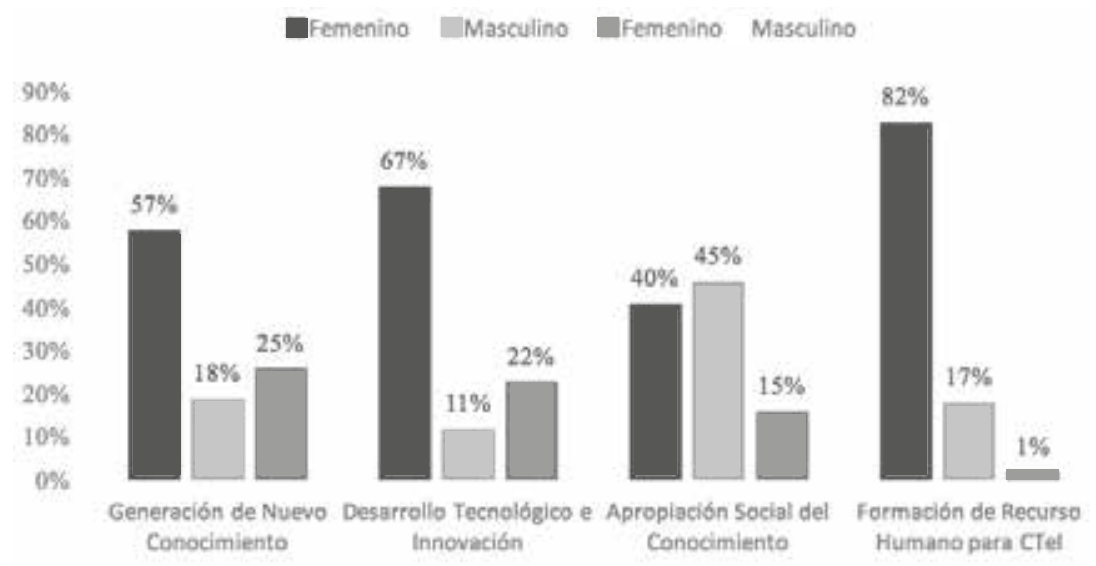

Figura 5. Producto de los grupos por género

Fuente: Elaboración propia, 2016.

Para finalizar, se totalizaron los datos como se muestra en la Figura 6, en la cual se expresa que el $74 \%$ de la producción de los grupos fue realizada por personas pertenecientes al género femenino, la producción masculina corresponde al $19 \%$, y por último, el $7 \%$ hace referencia a los productos realizados con participación de ambos géneros.

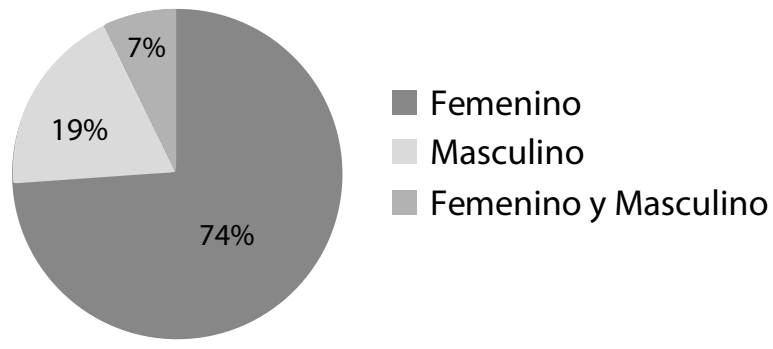

Figura 6. Producción total de los grupos por género

Elaboración propia, 2016.

De lo anterior se comprende que en los resultados de las revistas los hombres se encuentran dominantes en el proceso de producción académica; sin embargo, al revisar la producción de los grupos de investigación contable presentes en el Departamento del Quindío, se muestra que en el ejercicio de producción académica realizado en esta región las mujeres son dominantes. 


\section{Análisis de las entrevistas}

Por último, en aras de darle una mayor profundidad a la investigación y con el propósito de comparar los resultados cuantitativos con las experiencias desde la subjetividad individual e igualmente para comprender si las condiciones y concepciones culturales sobre la feminidad y la masculinidad han cambiado, se realizaron dos entrevistas a mujeres Contadoras Públicas, con estudios posgraduales, que en la actualidad laboran como docentes e investigadoras en instituciones de educación superior; siendo estas mujeres reconocidas en el eje cafetero y en el ámbito nacional por sus labores académicas. Teniendo en cuenta que, a petición de las entrevistadas, sus nombres permanecerán en el anonimato, sus percepciones fueron clasificadas de la siguiente forma:

- M.V. Del Departamento del Quindío.

- E.G. De la ciudad de Bogotá D.C.

En ese sentido, los resultados de las entrevistas fueron catalogados en las siguientes categorías:

Tabla 3.

Categorías de las entrevistas

1 Conocimiento sobre los estudios de género.

2 Experiencias investigativas.

3 El papel de las mujeres en la investigación contable según el contexto colombiano.

4 Discriminación e invisibilización por razones de género.

5 Igualdad de género en la investigación contable colombiana.

6 Importancia de los estudios de género en la disciplina contable.

Elaboración propia, 2016.

\section{Conocimiento sobre los estudios de género}

Las dos personas entrevistadas argumentan poseer conocimiento sobre los estudios de género realizados en el campo de conocimiento contable tanto en el ámbito nacional como en el internacional, siendo estos resultados de los constantes cambios en los diferentes contextos en los cuales tiene presencia la disciplina y la profesión. 


\section{Experiencias investigativas}

Para ambas las experiencias en este aspecto han sido gratificantes; principalmente, porque les ha ayudado en su proceso de formación académico; segundo, porque han aumentado sus intereses en cuanto al enriquecimiento intelectual; $\mathrm{y}$, por último, la satisfacción recibida por los aportes realizados mediante la construcción de nuevo conocimiento en búsqueda de la transformación y mejora de la disciplina de la profesión contable.

\section{El papel de las mujeres en la investigación contable según el contexto colombiano}

Las dos mujeres aseveran que tanto hombres como mujeres realizan labores investigativas con el fin de contribuir al desarrollo de la disciplina contable. Sin embargo M.V. afirma que la sociedad se encuentra en constante transformación y es necesario desde lo contable adaptarse a estos fenómenos sociales; a causa de ello expresa que existen contabilidades emergentes y en los espacios en los cuales se discuten las metodologías de medición, valoración, reconstrucción $\mathrm{y}$ transformación, se ve una gran participación femenina. Por otro lado ambas afirman que en la actualidad es muy difícil aplicar a convocatorias de Colciencias, ya que estas no van dirigidas a este campo de conocimiento.

\section{Discriminación e invisibilización por razones de género}

En esta sección las dos personas entrevistadas manifestaron ser víctimas de discriminación por el hecho de ser mujeres, sin embargo estas actividades discriminatorias se han exteriorizado en contextos diferentes. E.G. afirma que al momento de realizar investigaciones y documento académicos en compañía de hombres, las personas ajenas al trabajo asimilan que la labor importante fue realizada por el hombre y la mujer solo posee un papel de asistente en el proceso. Igualmente, M.V. manifiesta que tanto en los grupos de investigación como en los puestos administrativos de las instituciones universitarias se evidencian actividades excluyentes frente a las mujeres; en ese sentido relata que existen mujeres que desarrollan trabajos investigativos de gran importancia, pero al no poseer el título de líder de investigación quedan en estas publicaciones como coautoras siendo ellas las que los producen. También expresa que existen mujeres que pueden aspirar y tienen las capacidades para ejercer cargos directivos, aunque por ser consideras una amenaza para los directivos actuales se ejerce presión sobre ellas para que abandonen las instituciones, con la intención de negarles el protagonismo que les corresponde. 


\section{Igualdad de género en la investigación contable colombiana}

Ambas expresan que no existe igualdad de género en la investigación contable colombiana, teniendo en cuenta que aún existen las concepciones culturales en las cuales las mujeres poseen incapacidades intelectuales y físicas para realizar ciertas actividades consideradas de gran importancia. Por esto, es importante reconocer que esa igualdad se sigue buscando en los diferentes contextos sociales.

\section{Importancia de los estudios de género en la disciplina contable}

Las entrevistadas afirman que es importante realizar estudios de género en el campo de conocimiento contable, como respuesta a los cambios que se han manifestado tanto en lo académico como en la práctica de lo contable; un ejemplo de esto es la tendencia a la feminización de la profesión, cuando hace algunos años esta se reconocía por ser netamente masculina. En ese aspecto, es importante reconocer los aportes que las mujeres han venido realizando en pro del desarrollo y construcción de la disciplina contable, con el fin de darles el protagonismo que les corresponde.

El resultado de las entrevistas manifiesta que a pesar de la gran influencia femenina en el accionar contable aún siguen existiendo hechos discriminatorios y desiguales frente a las mujeres, tanto en el ejercicio académico como en el profesional; por tanto, se ve la necesidad de seguir desarrollando este tipo de estudios, con el propósito de reconocer los aportes de las mujeres y buscar esa igualdad y equidad tan anhelada.

\section{Discusión final}

Los resultados de esta investigación muestran la necesidad de seguir elaborando proyectos de investigación que aborden estas temáticas con la intención de continuar las discusiones en los diferentes contextos en los cuales se encuentra presente la disciplina y la profesión contable. Así mismo, expone diversos espacios en los cuales se manifiesta la implementación de las teorías feministas en el campo de conocimiento contable, como se muestra seguidamente:

Los estudios de género desarrollados por el ejercicio de la investigación contable utilizan metodologías que se caracterizan por ser incluyentes bajo las diferentes categorías sociales que rodean los ambientes humanos como: raza, etnia, sexo, género, entre otras. Dando reconocimiento a las actividades importantes realizadas tanto por hombres como por mujeres que le aportan al desarrollo de 
la disciplina y la profesión. Manifestando que la contabilidad posee género y refuerza los estereotipos plasmados sobre la feminidad y la masculinidad. En ese sentido, el número de publicaciones en las revistas seleccionadas expresan que aún existe una predominación masculina en la realización de este tipo de actividades académicas, aunque se reconoce la inclusión de las mujeres en estos espacios investigativos en los cuales hace algunos años no se les permitía participar o se les negaba el protagonismo por las labores realizadas.

Teniendo en cuenta que esta investigación se enfoca específicamente en la producción académica realizada en los grupos de investigación contable presentes en el Departamento del Quindío, categorizados desde una perspectiva de género, de ello se expresa una gran influencia y una amplia participación femenina en las labores investigativas, dando a entender en este aspecto que en comparación con los resultados de las revistas donde los hombres se manifiestan dominantes, a nivel de la región quindiana las mujeres son las que predominan en estos aspectos. Sin embargo, los resultados cuantitativos se muestran positivos en cuanto a la búsqueda de la igualdad y la equidad entre los géneros; no obstante, esto no implica que las concepciones culturales y los estereotipos sobre la feminidad y la masculinidad hayan cambiado.

En ese sentido, los resultados de las entrevistas exponen desde las experiencias de las entrevistadas que, aunque se han abierto hacia las mujeres diferentes espacios como la academia, siguen existiendo las manifestaciones de desigualdad y discriminación presentes por razones de sexo/género en los diferentes contextos sociales, y es importante empezar a reconocer estos acontecimientos con el propósito de seguir buscando la igualdad y equidad social.

Por lo dicho anteriormente, es importante la elaboración de investigaciones con estas perspectivas con la intención de dar reconocimiento a las labores de mujeres y hombres en el ejercicio profesional contable y en la construcción de conocimiento, e, igualmente, a este tipo de estudios desarrollados en el campo de conocimiento contable no se les ha asignado una importancia significativa y no se les ha transmitido un análisis suficiente, reconociendo que, estas percepciones pueden exponer y explicar los diferentes fenómenos sociales desde enfoques alternativos a los tradicionales. En ese aspecto se propone la realización de proyectos de investigación similares a este o la realización de nuevas investigaciones que le aporten al crecimiento del campo de conocimiento contable en estas temáticas. 


\section{Referencias}

Acosta, M., Patiño R., Valero, G. y Díaz, M. (2015). Las problemáticas de género: una introducción para su aplicación en estudios de contabilidad. Activos, 25, 33-72.

Bartra. E. (2012). Acerca de la investigación y la metodología feminista. En Blazquez, N; Flores, $F \&$ Ríos, M. (Coordinadoras). Investigación feminista: epistemología, metodología y representaciones sociales. Ciudad de México, México: Universidad Nacional Autónoma de México, Centro de Investigaciones Interdisciplinarias en Ciencias y Humanidades: Centro Regional de Investigaciones Multidisciplinarias: Facultad de Psicología.

Blazquez. N. (2012). Epistemología feminista: temas centrales. En Blazquez, $N$; Flores, $F$ \& Ríos, M. (Coordinadoras). Investigación feminista: epistemología, metodología y representaciones sociales. Ciudad de México, México: Universidad Nacional Autónoma de México, Centro de Investigaciones Interdisciplinarias en Ciencias y Humanidades: Centro Regional de Investigaciones Multidisciplinarias: Facultad de Psicología.

Bonilla, E. (1993). La mujer colombiana en la universidad y en el mundo del trabajo. Bogotá, Colombia: Universidad de los Andes.

Cabral, B y García, C. (s.f). El género. Una categoría de análisis crítico para repensar las relaciones sociales entre los sexos. Recuperado de http:// www.saber.ula.ve/bitstream/123456789/16344/1/genero-categoria.pdf

Calvo, A. (2010). Débito y crédito contable... algo más que un saldo sociología de la profesión contable en Colombia (Tesis de maestría). Bogotá, Colombia: Universidad Nacional de Colombia.

Cooper, C. (2001). From women's liberation to feminism: reflections in accounting academia. Accounting Forum, 25, 214-145.

Cubides, H. (1999). Evolución de la capacitación y formación de los contadores públicos. En Historia de la Contaduría Pública en Colombia Siglo XX. Bogotá, Colombia: Fundación Universitaria Central. 
Departamento Administrativo de Ciencia, Tecnología e Innovación COLCIENCIAS. (2016). Publicación de resultados finales de la convocatoria 737 de 2015. Recuperado de http://www.colciencias.gov. $\mathrm{co} / \mathrm{sites} /$ default/files/listado-publicacion-resultadosfinales-conv737gruposinvestigacion-consulta.pdf

Departamento Administrativo de Ciencia, Tecnología e Innovación COLCIENCIAS. (2015). Modelo de medición de grupos de investigación, desarrollo tecnológico o de innovación y de reconocimiento de investigadores del sistema nacional de ciencia, tecnología e innovación, año 2015. Recuperado de http://www.colciencias.gov.co/sites/default/ files/upload/noticias/mediciondegrupos-actene2015.pdf

Facio, A. y Fries, L. (1999). Feminismo, género y patriarcado. En A. Facio, y L. Fries (Editores), Género y Derecho (pp. 6-38). Santiago de Chile, Chile.

Haynes, K. (2008a). (Re)figuring accounting and maternal bodies: The gendered embodiment of accounting professionals. Accounting, Organizations and Society, 33, 328-348.

Haynes, K. (2008b). Transforming identities: Accounting professionals and the transition to motherhood. Critical Perspectives on Accounting, 19, 620-642.

Haynes, K. (2016). Accounting as gendering and gendered: A review of 25 years of critical accounting research on gender, Critical Perspectives on Accounting. Recuperado de http://dx.doi.org/10.1016/j.cpa.2016.06.004

Junta Central de Contadores. (2016). [Comunicación personal, Mayo 3 de 2016].

León, F y Naranjo, Y. (2011). Profesionales de contaduría pública de Bogotá: trayectorias y prácticas. Recuperado de https://revistas.lasalle.edu.co/ index.php/te/article/view/452/373.

Méndez, M. (2010). De la contabilidad doméstica a la profesionalización contable de las mujeres en el Siglo XIX. Mujeres y Economía, 852, 91-98.

Nuño. T. (2000). Género y ciencia. La educación científica. Revista de psicodidáctica, 183-214. 


\section{Contexto Losor. E.}

Riegraf, B \& Aulenbacher, B. (2012). Investigación feminista - ¿quo vadis? Recuento metodológico histórico y perspectiva epistemológica a futuro. En Garza, E \& Leyva, G. Eds. Tratado de Metodología de las ciencias sociales: perspectivas actuales. 534-553.

Ríos. M. (2012). Metodología de las ciencias sociales y perspectiva de género. En Blazquez, N; Flores, $F$ \& Ríos, M. Coordinadoras. Investigación feminista: epistemología, metodología y representaciones sociales. Ciudad de México, México: Universidad Nacional Autónoma de México, Centro de Investigaciones Interdisciplinarias en Ciencias y Humanidades: Centro Regional de Investigaciones Multidisciplinarias: Facultad de Psicología.

Rodríguez, M. (2014). Mujeres las voces desobedientes de nuestra historia: notas para develar la potencialidad de los estudios de género en el ámbito contable. Revista Adversia, 15. Recuperado de https://aprendeenlinea. udea.edu.co/revistas/index.php/adversia/article/view/20908

Todaro, R. y Rodríguez, R. (2001) Ampliar los límites de la economía. En Todaro, $\mathrm{R} \&$ Rodríguez, R, eds. El género en la economía. Santiago de Chile.

\section{Para citar este artículo:}

López, É. (2017). Análisis del papel de las mujeres en la investigación contable desarrollada en el Departamento del Quindío desde una perspectiva de género. En-Contexto, 6(9), 157-180. 